\title{
Hagiografía, milagros y providencia: fundamentos teológico- históricos de la Historia Francorum de Gregorio de Tours (siglo VI)
}

\author{
Marcelo E. Aguirre Durán \\ INSTITUTO DE HISTORIA \\ UNIVERSIDAD DE LOS ANDES
}

\section{LA FIGURA DE SAN GREGORIO}

San Gregorio de Tours, de nombre Georgius Florentinus Gregorius, nació en el seno de una familia de la aristocracia galorromana, tanto por parte paterna como materna ${ }^{1}$, en el año 538 ó $539^{2}$. Natural de Clermont, en la región de Auvernia ${ }^{3}$, su origen senatorial le hizo recibir una cultura y educación latinas, aunque su lengua materna era el latín vulgar, ya en lenta evolución ${ }^{4}$.

Una vez muerto su padre, Florentius, aproximadamente a la edad de ocho años, su madre Armentaria habría encargado su educación a su tío-abuelo materno y futuro obispo de Lyon, san Nizier. A esa edad habría comenzado a leer y a escribir ${ }^{5}$. Posteriormente, habría pasado a la tutela de su tío paterno Gallus, obispo de Clermont. Sus estudios habrían sido fundamentalmente eclesiásticos, con especial hincapié en las

1 CH. LELONG, Grégoire de Tours. Sa vie et son auvre (Chambray-lès-Tours 1995) 1415. Esta biografía presenta esquemáticamente los árboles genealógicos de los padres de san Gregorio. Es el estudio biográfico más actual que conocemos sobre nuestro autor.

2 L. PIETRI, "Grégoire de Tours (saint)» en Dictionnaire de spiritualité ascétique et mystique: doctrine et histoire, vol. VI (Paris 1967) col. 1020.

3 Región francesa (Auvergne) ubicada en el centro sur del país. Su capital es Clermont-Ferrand.

$4 \quad$ R. LATOUCHE (ed.), Grégoire de Tours. Histoire des francs, vol. I (Paris 1963) 6.

5 M. BOnNET, Le latin de Grégoire de Tours (Hildesheim 1968) 48. 
Sagradas Escrituras, pero sin mucho conocimiento de los Santos Padres y del mundo clásico ${ }^{6}$.

Su tío Gallus y su preceptor Avito se podrían ver como los dos maestros de Gregorio, y a ellos debería su cultura y el acercamiento a las letras. Estudió en la Schola Episcopal de Clermont, lugar en donde se impartía únicamente la Sacra Pagina, es decir, las letras latinas a partir de los textos de las Sagradas Escrituras ${ }^{7}$.

Aunque el historiador belga Godefroid Kurth afirma que es en esta escuela en donde Gregorio debió haber conocido y se debió haber familiarizado con algunas letras de la Antigüedad pagana, lo cual podría darse como un elemento independiente de la barbarie de su lenguaje escrito $^{8}$, es probable que su conocimiento de los autores clásicos no fuera destacado.

No resultaría extraño pensar que las obras de gramática y los autores antiguos le fueran más bien desconocidos, carencia que más tarde habría intentado remediar leyendo, por ejemplo, a Virgilio9. De esta manera, es posible que hubiese llenado algunas lagunas en relación a autores profanos tales como Salustio, Horacio y Plinio ${ }^{10}$, aunque él tendría clara conciencia de que escribía en un latín vulgar, simple y directo ${ }^{11}$, privilegiando el mayor alcance de su obra a personas menos letradas ${ }^{12}$.

Hacia el 563 fue ordenado diácono. Poco después habría caído gravemente enfermo y habría peregrinado a Tours para obtener la curación

6 C. Moreschini - E. Norelli, Historia de la literatura cristiana antigua griega y latina. Desde el concilio de Nicea hasta los comienzos de la Edad Media, vol. II (Madrid 2007) 594; L. PIETRI, "Grégoire de Tours (saint)», col. 1020.

7 P. RICHÉ, Éducation et culture dans l'Occident barbare. VIe - VIIIe siècles (Paris 1962) 159.

8 G. KURTH, «Saint Grégoire de Tours et les études classiques au VIe siècle», en Revue des questions historiques 24 (1878) 588.

$9 \quad$ P. RicHÉ, Éducation et culture dans l'Occident barbare, 235.

10 L. PIETRI, «Grégoire de Tours (saint)», col. 1020.

11 J. M. WALLACE-HADRILL, «The Work of Gregory of Tours in the Light of Modern Research", en The Long-Haired Kings and Others Studies in Frankish History (London 1962) 50; H. PIRENNE, Historia de Europa: desde las invasiones hasta el siglo XVI (México D. F. 1956) 42. El mismo Gregorio lo dice: GREgOrIO DE TOURS, Historia Francorum, I, Praefatio: loquentem rusticum multi.

12 Cf. Gregorio de Tours, Historia Francorum, I, Praefatio. 
milagrosa por intercesión de san Martín ${ }^{13}$. Una vez sanado a través de la acción sobrenatural atribuida a este santo, permaneció algún tiempo en dicha ciudad -magnífico puesto de observación entre el norte y el sur de Francia ${ }^{14}$ - recibiendo en el año 573 el episcopado turonense, sucediendo al obispo Eufronio, su tío-abuelo materno ${ }^{15}$. Murió, probablemente, el 17 de noviembre del 594 .

Gabriel Monod, por su parte, ha visto en el obispo de Tours un hombre que: «no ha podido escapar de la decadencia intelectual de toda su época» ${ }^{16}$, palabras que, de cierta forma se verían reflejadas en el propio Gregorio, quien al comenzar su Historia Francorum ${ }^{17}$ escribe: «el estudio de las bellas letras está en decadencia y, más bien, él se muere en las ciudades de la Galia» ${ }^{18}$.

En tanto que hijo de una familia patricia, su vida se habría desarrollado dentro de un ambiente enteramente romano ${ }^{19}$. Siendo educado bajo el latín de la Auvernia, este no sería solo una lengua literaria, sino que sería, también, su lengua materna ${ }^{20}$, lengua propia del ambiente católico en el que se desarrolló su educación.

Como ha sido señalado por Pierre Riché, la figura del obispo de Tours no podría entenderse fuera del ambiente romano, ya que los propios francos se referían a los aquitanos, entrado ya el siglo VIII, con el nombre de romani ${ }^{21}$. No sería este un detalle menor, ya que la región que vio nacer a Gregorio habría sido una importante defensora del «patriotismo

13 R. Latouche, «Un évêque historien: Grégoire de Tours» en Gaulois et francs. De Vercingétorix à Charlemagne (Paris - Grenoble 1965) 254.

14 N. COHN, En pos del milenio. Revolucionarios, milenaristas y anarquistas misticos de la Edad Media (Madrid 1983) 40.

15 CH. LELONG, Grégoire de Tours, 14.

16 G. MONOD, Études critiques sur les sources de l'histoire mérovingienne, I parte (Paris 1872) 119-120.

17 Para el texto latino usaremos la edición de la Patrología de Migne: PL 71, Historiae Francorum Libri Decem, aunque seguiremos la numeración de la edición de Robert Latouche editada en Les Belles Lettres.

18 Gregorio de Tours, Historia Francorum, I, Praefatio: decedente atque immo potius pereunte ab urbibus Gallicanis liberalium cultura litterarum.

19 G. KURTH, Études franques, vol. II (Paris - Bruxelles 1919) 118.

20 M. Bonnet, Le latin de Grégoire de Tours, 28.

21 P. RICHÉ, Éducation et culture, 226. 
galorromano ${ }^{22}$, origen que nuestro obispo no habría olvidado en tanto que galo de la Aquitania ${ }^{23}$.

También resulta importante destacar que el cristianismo heredado por Gregorio sería un cristianismo que ya había penetrado profundamente en la sociedad galo-romana, constituyendo una suerte de nueva romanidad, y que sería una clave interpretativa para entender el relato histórico de la Historia Francorum. En definitiva, el santo obispo de Tours sería hijo de una Galia convertida a un cristianismo profundo que, en poco tiempo, le habría dado una característica particular y distintiva ${ }^{24}$.

La obra del prelado turonense se podría entender bajo el modelo de las nuevas historias nacionales, las cuales, de forma llamativa, fueron hechas por hombres romanos en cuanto a cultura ${ }^{25}$, como es también el caso de san Isidoro de Sevilla. En este aspecto la narración que relata la historia del pueblo franco tendría el sentido universal de la historiografía cristiana y, al mismo tiempo, apuntaría a la particularidad del mundo bárbaro.

Uno de los rasgos que posiblemente más llama la atención en el obispo de Tours es su marcada y estricta ortodoxia católica, la cual deja en evidencia a lo largo de toda su obra. Esta ortodoxia se debería, probablemente, a la influencia y al ambiente familiar en el que le habría tocado desenvolverse, ambiente que se podría definir como el de las típicas familias mitradas, ya que Gregorio tenía como antepasados a mártires y numerosos obispos ${ }^{26}$. Esto marcaría, por tanto, su visión ideológica e histórica ${ }^{27}$.

22 R. LATOUCHE, «Grégoire de Tours et les premiers historiens de la France» en Lettres d'humanité, vol. II (Paris 1943) 83.

23 S. TeILLET, Des goths à la nation gothique. Les origines de l'idée de nation en Occident du Ve au VIIe siècle (Paris 1984) 374.

24 J. FONTAINE, «L'apport du christianisme à la prise de conscience de la 'patrie gauloise' sous la dynastie theodosienne» en La patrie gauloise d'Agrippa au VIe siècle (Lyon 1983) 200.

25 A. MOMigLiano, «L'età del trapazo fra storiografia antica e storiografia medievale (320-550 D.C.)» en La storiografia altomedievale, vol. I (Spoleto 1970) 116.

26 Ch. Lelong, Grégoire de Tours, 17.

27 M. A. Rodríguez de LA PEÑA, Los reyes sabios. Cultura y poder en la Antigüedad tardía y la alta Edad Media (Madrid 2008) 319. 
La producción literaria de nuestro autor es numerosa, y de ella deja constancia en el capítulo XXXI del libro X de su Historia Francorum ${ }^{28}$. Además de la obra recién mencionada, que consta de diez libros, escribió siete libros de milagros (Septem Libri Miraculorum), uno sobre la vida de los Padres desde los siglos IV al VI (Liber Vitae Patrum), un comentario a los salmos (Psalterii Tractatus), y un tratado sobre las estrellas, con el fin responder a algunas cuestiones litúrgicas (De Cursu Stellarum Ratio).

La Historia Francorum ha pasado a la posteridad como la primera obra de la historiografía francesa. En ella encontramos, en primer lugar, una historia universal ${ }^{29}$, que va desde la creación del mundo hasta el año 591. Dividida en diez libros, a partir del libro II el autor comienza a narrar hechos cada vez más particulares y, por tanto, de un interés cada vez más local.

Aunque resulta más bien paradójico, el relato histórico de Gregorio de Tours sería una descripción de un galo-romano sobre el mundo germánico, pero no se encontraría en él una penetración en la mentalidad de este último. Tal como ha escrito Riché, su historia sería una historia eclesiástica de los francos, pero el mundo bárbaro estaría reducido a lo que se relacionaría directamente con este objetivo ${ }^{30}$.

Estaríamos, entonces, ante una historia que comienza con un relato del pueblo de Dios, pero que luego introducirá la novedad de ver a este pueblo concretado en la situación particular de la Galia, bajo la dirección de sus obispos ${ }^{31}$. Esto se vería acentuado a partir del libro V, en donde la obra adquirirá una estructura de informativo de los diversos hechos y anécdotas acontecidos ${ }^{32}$.

Tradicionalmente se piensa que la Historia Francorum habría sido redactada entre los años 574-575 y 591, aunque es probable que el autor

28 Gregorio De Tours, Historia Francorum, X, XXXI: decem libros Historiarum, septem Miraculorum, unum de Vita Patrum scripsi; in Psalterii tractatu librum unum commentatus sum; de Cursibus etiam ecclesiasticis unum librum condidi.

29 R. Latouche (ed.), Grégoire de Tours, 8.

30 P. RICHÉ, Éducation et culture, 226.

31 E. Mitre FERnÁNDEZ, «Historia y pensamiento histórico en la transición al medioevo" en Historiografía y mentalidades históricas en la Europa medieval (Madrid 1982) 55; H. PIRENNE, Historia de Europa, 42.

32 R. Latouche (ed.), Grégoire de Tours, 9. 
haya revisado varias veces los distintos libros. Por su parte, en la última edición bilingüe latín-italiano, editada en Nápoles por Massimo Oldoni, su redacción es datada novedosamente entre los años 576 al 593, como se detalla a continuación ${ }^{33}$ : libros I al IV, desde Adán hasta el 575, escritos entre el 576 y el 580; libro V, desde el 575 al 580, escrito en el 581; libros VI y VII, desde el 581 al 585, escritos entre el 582 y el 586; libro VIII, desde el 585 al 587, escrito en el 589-590; libro IX, desde el 587 al 589, escrito en el 589-590; libro X, desde el 589-591, escrito desde el 590 al 593.

Aunque es una historia de la monarquía franca ${ }^{34}$, san Gregorio tendría la intención de unir su obra con la historia universal, y para ello se servirá de la Crónica y de la Historia Eclesiástica de Eusebio de Cesarea, traducidas al latín por san Jerónimo y Rufino, respectivamente. También utilizará la continuación cronística hecha por el santo de Estridón, y a ello le sumaría, principalmente, las Historiarum Adversum Paganos del hispano Paulo Orosio. También habría trabajado con algunos historiadores del siglo $\mathrm{V}$, así como con algunos escritores cristianos, tal como lo afirma Charles Lelong al decir que: «el obispo turonense era gran lector de Sulpicio Severo" ${ }^{35}$.

\section{LA HAGIOGRAFÍA Y LOS MILAGROS}

Gregorio no es ni un teólogo ${ }^{36}$ ni un exegeta ${ }^{37}$, y eso se nota en su obra. Su conocimiento de san Agustín y de san Jerónimo, por ejemplo, sería limitado ${ }^{38}$. Aunque comienza con una profesión de fe, esta tendría como objetivo demostrar el radical rechazo a todo tipo de herejía, en particular el arrianismo ${ }^{39}$. El dogma de la Santísima Trinidad es el punto de partida de su profesión, marcadamente nicena, la cual desea explicitar claramen-

33 M. OldONI (ed.), Storia dei franchi, vol. I (Napoli 2001) XXXII.

34 F. L. GANSHOF, «L'historiographie dans la monarchie franque sous les mérovingiens et les carolingiens», en La storiografia altomedievale, vol. II (Spoleto 1970) 633.

35 Ch. Lelong, Grégoire de Tours, 33.

36 G. VInAY, San Gregorio di Tours (Torino 1940) 22.

37 L. PIETRI, «Grégoire de Tours (saint)», col. 1021.

38 Ch. Lelong, Grégoire de Tours, 76.

39 L. PIETRI, «Grégoire de Tours (saint)», col. 1021. 
te para no confundir al lector: "yo deseo confesar mi fe para que aquel que me lea no dude de que yo soy católico» ${ }^{40}$.

En este sentido, la confirmación pública de su fe le permitiría tener la autoridad necesaria para poder escribir una historia del pueblo franco en la que el carácter edificante tendría cierto lugar ${ }^{41}$. Este carácter edificante tendría un sentido moralizador, aunque no tan explícito como en las obras históricas de otros autores (Salviano o Sulpicio, por ejemplo), sino que aquí se unirían una preocupación por las costumbres, con el simple hecho de narrar copiosamente una serie de detalles, por el solo gusto de contar ${ }^{42}$. Para ello incluiría, en un sentido casi pastoral y didáctico ${ }^{43}$, relatos hagiográficos y testimonios de fe. Quizás es en esto en donde Reydellet ha querido ver un valor moralizante, a veces evidente y a veces oculto ${ }^{44}$.

La hagiografía sería una de las claves para poder entender este sentido edificante en la obra de Gregorio. Ella sería el lugar en donde el Turonense encontraría los fundamentos de una narrativa religiosa y, en cierta medida, le permitiría mostrar figuras fieles al dogma, marcadas por una cierta heroicidad que se verá demostrada en el triunfo de la verdadera fe en un ambiente dominado por la barbarie ${ }^{45}$.

El Turonense no cita menos de treinta y cinco vidas de santos, lo cual indicaría que habría conocido de manera importante la literatura hagiográfica que tenía a su alcance ${ }^{46}$. Esto no debería ser pasado por alto, pues sería una prueba de la importancia dada por el autor a las vidas

40 Gregorio De ToUrs, Historia Francorum, I, Prólogo: prius fidem meam proferre cupio, ut qui ligirit me non dubitet esse catholicum.

${ }_{41}$ M. REYDELLET, La royauté dans la littérature latine de Sidoine Apollinaire à Isidore de Séville (Roma 1981) 364-365; L. HALPHEN, "Grégoire de Tours. Historien de Clovis» en Mélanges d'histoire du Moyen Âge. Offerts à M. Ferdinand Lot (Paris 1925) 240. Reydellet sostiene que en el libro V los capítulos 18-20 y 38-39 forman una unidad edificante para el lector.

42 R. LATOUCHE, "Quelques réflexions sur la psychologie de Grégoire de Tours», Le Moyen Âge. Revue d'histoire et de philologie, LXIX (1963) 15.

43 W. GOFFART, The Narrators of Barbarian History (A. D. 550-800). Jordanes, Gregory of Tours, Bede and Paul the Deacon (New Jersey 1988) 182.

44 M. REYDELLET, La royauté dans la littérature latine, 364.

45 E. Mitre FernándeZ, Historia y pensamiento histórico. Estudio y antología (Madrid 1997) 37.

46 G. KURTH, Études franques, 136. 
ejemplares y a los relatos de milagros, en tanto que fuentes históricas y modelos de vida cristiana.

Como ha señalado Wallace-Hadrill, la hagiografía sería uno de los elementos principales de su actividad literaria ${ }^{47}$. De esa forma, una de las principales figuras cristianas tomadas por el obispo de Tours sería la de su antecesor san Martín. Para él este sería su propio modelo de obispo, defensor de la fe y enemigo de los herejes, tal como lo escribe al comienzo de su obra: «destruyó templos, destruyó la herejía, edificó iglesias» ${ }^{48}$.

Los milagros de san Martín resultan numerosos y, en cuanto protector de la diócesis de Tours, aparecerán en la Historia Francorum de forma aún más destacada ${ }^{49}$. De algún modo la serie de milagros de este santo le otorga el matiz pastoral y edificante al relato histórico escrito por Gregorio, el cual convivirá paralelamente, sin muchas conexiones evidentes, con el interés en la historia de los reyes francos de su tiempo. La figura de san Martín, en la concepción de nuestro autor, haría de su diócesis un lugar de peregrinación y de múltiples milagros, tomando incluso el valor de capital religiosa de la Galia ${ }^{50}$.

En Gregorio la conciencia de ser sucesor en la sede episcopal del santo obispo de Tours sería tan fuerte que marcaría toda su carrera eclesiástica, toda su labor apostólica y su actividad evangelizadora ${ }^{51}$. Todo lo relacionado con su santo predecesor tendría una connotación de sagrado, manifestado incluso en el deseo de emular su estilo de vida ascética ${ }^{52}$, lo cual no solo sería visto por el Turonense, sino que también por los hombres de su tiempo ${ }^{53}$.

47 J. M. WALLACE-HADRILL, «The Work of Gregory of Tours» 54-55.

48 Gregorio De Tours, Historia Francorum, I, 39: hic enim fana distruxit, heresem oppraessit, eclesias aedificavit.

49 F. L. GANSHOF, «L'historiographie dans la monarchie franque», 635.

50 C. MORESCHINI - E. NORELlI, Historia de la literatura cristiana antigua griega y latina, 595-596; CH. LELONG, Grégoire de Tours, 33-35.

51 J. M. WALLACE-HADRILL, "The Work of Gregory of Tours» 52.

52 M. REYDELLET, «Pensée et pratique politiques chez Grégoire de Tours» en Gregorio di Tours. Convegni del centro di studi sulla spiritualità medievale (Perugia 1977) 177.

53 Véase. R. A. MEUNIER, Grégoire de Tours et l'histoire morale du centre-ouest de la France. Étude sur les manifestations des liaisons spirituelles régionales entre Loire et Gironde, des confins d'Auvergne à l'océan, au dernier quart du VIe siècle (Poitiers 1946) 27-36. 
Ahora bien, el mundo de los milagros no sería una realidad lejana para el obispo historiador, sino que este formaría parte de lo más propio del mundo en el que le habría tocado vivir y, de esta forma, lo plasmará en su obra histórica, es decir, como un hecho normal y cotidiano, válido para la interpretación de la historia como un hecho más ${ }^{54}$.

Por lo tanto, creemos que no se podría hablar de una ingenuidad en Gregorio sino que, más bien, habría que entender que su creencia en los acontecimientos sobrenaturales le sería común con la creencia de los hombres de su tiempo ${ }^{55}$. A esto se sumaría el hecho de haber nacido bajo un ambiente "extremadamente devoto ${ }^{56}$, dentro del cual lo prodigioso tendría una clara explicación en lo sobrenatural.

La hagiografía ocupa un lugar tan preponderante en su obra que esto lleva a pensar, consecuentemente, que el culto a los santos podría entenderse como un elemento principal dentro de su religiosidad. Este culto le permite observar la historia salutis a través de las figuras de los elegidos, de manera particular san Martín, quien entraría dentro del modelo de una tipología profética ${ }^{57}$, tal como aparecería también en Sulpicio Severo.

Para Massimo Oldoni, en una afirmación un tanto arriesgada y a nuestro juicio errónea, el lugar de la hagiografía en la obra del Turonense sería tan importante, que esta reemplazaría incluso el significado y la religiosidad que debería tener la Sagrada Escritura ${ }^{58}$.

Ante esto resulta necesario recordar que la religiosidad del obispo de Tours, como la de cualquier pensador cristiano de la época, radicaría en una fidelidad y centralidad en la Sagrada Escritura. En tanto que Palabra Revelada, Ella se constituye en el punto de partida de toda su reflexión y, a su vez, es la inspiración para su cercanía a los relatos hagiográficos,

54 W. GOFFART, The Narrators of Barbarian History, 131, 234; P. J. GALÁN SÁNCHEZ, El género historiográfico de la Chronica. Las crónicas hispanas de época visigoda (Cáceres 1994) 33.

55 R. Latouche, "Quelques réflexions sur la psychologie de Grégoire de Tours» 7.

56 CH. LELONG, Grégoire de Tours, 17.

57 B. DE GAIFFIER, «Hagiographie et historiographie» en La storiografia altomedievale, vol. I, (Spoleto 1970) 161.

58 M. Oldoni, "Gregorio di Tours e i 'Libri Historiarum': Letture e fonti, metodi e ragioni», en Studi medievali, II (1972) 610-612. 
en tanto que estos representarían una imitatio Christi. En ningún caso la hagiografía se encontraría separada de un fundamento bíblico anterior $^{59}$, y tampoco podría constituir lo que ha sido llamado «la formidable dimensión del sentido humano de la religiosidad ${ }^{60}$, pues ella se encontraría, más bien, dentro de su preocupación moral.

Por su parte, y muy acertadamente, Paul Antin ha expresado que: «la Biblia es para el cristiano mucho mejor que, para el pagano, Homero o Virgilio o Cicerón. Para Gregorio, letrado modesto y cristiano convencido, el recurso frecuente en la expresión bíblica es una manera de vivir en presencia de Dios» ${ }^{61}$.

Por consiguiente, si bien la hagiografía sería una de las principales fuentes de la obra del obispo de Tours, no podríamos llegar a afirmar que esta reemplazaría el valor incluso de la Sagrada Escritura, pues él logra distinguir las funciones del historiador y del hagiógrafo, y utiliza esta última para dar elementos que el trabajo puramente histórico no le permite ${ }^{62}$.

De este modo, el género hagiográfico estaría al servicio de un relato mayor, pues el cumplimiento de las profecías bíblicas en la figura de Jesucristo vendría a ser el hecho teológico por excelencia y, asimismo, representaría para la historia el prototipo de vida de los santos, lo cual se explicaría siempre dentro de su preocupación por la instrucción moral y religiosa ${ }^{63}$.

Gregorio tendría necesidad de modelos de vida moral cristiana, sobre todo en la medida en que se constituiría en narrador del tiempo de los reyes francos ${ }^{64}$, pero esto no implicaría, necesariamente, una contraposición con los relatos bíblicos. Si bien los pasajes bíblicos no aparecerán anotados y parafraseados permanentemente y de manera explícita, como en el caso de otros autores cristianos por él trabajados, sí se puede notar que la vida de los santos resulta ser un reflejo de la Escritura, ya que: «él

59 P. ANTIN, «Emplois de la Bible chez Grégoire de Tours et Mgr Pie», en Latomus, XXVI (1967) 780.

60 M. OldONI, «Gregorio di Tours e i 'Libri Historiarum'», 610.

61 P. ANTIN, «Emplois de la Bible chez Grégoire de Tours et Mgr Pie», 780-781.

62 J. M. WALlaCe-HadRILL, "The Work of Gregory of Tours», 54.

63 Cf. W. GOFFART, The Narrators of Barbarian History, 204-205.

64 J. M. WALLACE-HADRILL, "The Work of Gregory of Tours», 61. 
pensaba que su prosa era bella y cuidada en la medida en que ella estaba centrada en la Escritura» ${ }^{65}$.

Ahora bien, en cuanto a los milagros, la creencia aparente por parte de Gregorio habría que entenderla dentro de la cosmovisión de un mundo sobrenatural que actuaría, de alguna forma, en lo terrenal. En palabras de Kurth: «se puede decir que Gregorio no conoce nada más natural que lo sobrenatural ${ }^{66}$, lo cual también sería predicable de cualquier hombre medieval. En este sentido, el milagro viene a ser la prueba de la elección por parte de Dios de algunos hombres, los cuales distribuyen, por designio divino, las recompensas y penitencias de este mundo ${ }^{67}$.

En la Historia Francorum los milagros y diferentes portentos obtendrían una connotación particular. En mayor o menor medida estos corresponderían a narraciones verdaderas que el autor no se cuestiona; le servirían como una fuente histórica y, al mismo tiempo, mostrarían las relaciones entre los hombres comunes y los santos, en tanto que elegidos de Dios ${ }^{68}$.

\section{III. ¿ES LA HISTORIA FRANCORUM UNA OBRA PROVIDENCIALISTA?}

La dualidad Bien/Mal desarrollada, por ejemplo, en los relatos históricos de Orosio, Salviano y Sulpicio, tendrían directa relación con la acción de Dios a través de su Providencia. En este sentido, no sería difícil pensar que este esquema habría sido adoptado sin mayores complicaciones por san Gregorio de Tours, aunque sus características le darían particularidades que incluso podrían hacer pensar en una visión mucho más práctica y real, sin un trasfondo mayormente teológico ${ }^{69}$.

Los esquemas de los pensadores cristianos de la historia, a partir de Eusebio de Cesarea, fueron aplicados en estas primeras historias que se entendieron como nacionales, pues su interés era, principalmente, el de explicar la presencia de la fe en medio de pueblos dominados por la barbarie, la herejía o el paganismo ${ }^{70}$. El obispo turonense, en cierta medida, no sería una excepción a esta continuidad histórica.

\footnotetext{
65 P. Antin, «Emplois de la Bible chez Grégoire de Tours et Mgr Pie», 780.

66 G. KURTH, Études franques, 122.

67 R. LATOUCHE (ed.), Grégoire de Tours, 14.

68 W. GOFFART, The Narrators of Barbarian History, 131.

69 M. OldONI (ed.), Storia dei franchi, L.

70 E. Mitre FernándeZ, Historia y pensamiento histórico, 37.
} 
De esta manera, la acción de Dios en la historia se daría, tal como aparece en el libro I, a partir del deseo de Cristo de salvar a todos los pueblos, partiendo del mundo judío. Gregorio tendría la preocupación de hacer notar cómo el mensaje de salvación parte de un pueblo, pero se hace universal, alcanzando incluso la realidad que a él mismo le habría tocado vivir: «el mismo Señor, prometido por la voz de los patriarcas y de los profetas, descendido en el seno de la Virgen María por la acción del Espíritu Santo, se dignó nacer para la redención tanto de esta nación [judíos] como de todas las naciones» ${ }^{71}$.

Su trabajo de compilación de datos tendría un objetivo claro: la continuidad de la historia, tal como lo expresa al comienzo del Libro II: «de este modo Eusebio, Severo, Jerónimo así como Orosio han insertado en sus crónicas, a la vez, relatos de guerras de reyes y aquellos milagros de mártires. Es así que nosotros hemos compuesto nuestro escrito para que la enumeración del orden de siglos y el cálculo de años hasta nuestro tiempo sea más fácil de descubrir en su totalidad $»^{72}$.

En esa continuidad, la utilización de san Jerónimo se dará hasta el capítulo XLI del primer libro, lugar en donde se detiene y hace mención explícita a la lectura de las Historiarum Adversum Paganos, en donde escribe: «en este lugar se detiene Jerónimo, y a partir de esta época es Orosio presbítero quien ha escrito la siguiente» ${ }^{73}$. Quizás intentaría mostrarse él mismo como un continuador, adquiriendo la legitimación dada por la utilización y adopción de la idea providencial de la historia que habían plasmado en sus trabajos estos escritores.

Para muchos autores ${ }^{74}$ esta vinculación con obras fundamentales como las recién mencionadas sería una prueba más de su visión de la conducción, por parte de Dios, de la historia de los hombres. El desafío

71 Gregorio De Tours, Historia Francorum, I, 16: donec ipse Dominus patriarcharum prophetarumque vocibus repromissus, virginis Mariae utero per Spiritum sanctum inlapsus, ad redimptione nasci tam illius gentes quam omnium gentium dignaretur.

72 Gregorio de Tours, Historia Francorum, II, Prólogo: sic et Eusebius, Severus Hieronimusque in chronicis atque Horosius et bella regum et virtutes martyrum pariter texuerunt. Ita et nos idcircum sic scripsemus, quod facilius saeculorum ordo vel annorum ratio usque nostra tempora tota repperiatur.

73 Gregorio De TOURS, Historia Francorum, I, 41: hucusque Hieronimus; ab hoc vero tempore Horosius presbiter plus scripsit.

74 Bonnet, Kurth, Monod, Ganshof, Halphen, Latouche, entre otros. 
de la Providencia no estaría solo en la acción de los cristianos con los paganos (gentiles), sino que en el siglo VI esta estaría dada también por la lucha de cristianos contra paganos y herejes (bárbaros) ${ }^{75}$.

Sumado a lo anterior, su visión histórica adopta ciertas características apologéticas, como en Orosio, y en tanto que pastor de una comunidad católica, ve la necesidad inminente de reaccionar ante el peligro amenazante de la herejía, tal como lo expresa al comienzo de su prefacio general, escrito probablemente al final de sus días: «las iglesias eran atacadas por los heréticos y protegidas por los católicos» ${ }^{76}$.

Como bien ha apuntado Suzanne Teillet, el horror que al obispo de Tours le provocaría la herejía arriana, definida como: «aquella pérfida y deforme herejía ${ }^{77}$, le llevaría incluso a olvidar, de cierta forma, que los miembros de esta, aunque no católicos, sí tenían la condición de cristianos. Esto mostraría el total rechazo hacia la herejía, y se vería reflejado en la oposición de los términos haeretici y christiani, términos para él totalmente contrarios y distintos ${ }^{78}$.

Aunque Gregorio no es un teólogo ${ }^{79}$, como lo hemos mencionado más arriba, la preocupación por el arrianismo se manifiesta a lo largo de toda su obra. Él logrará ver sus implicaciones doctrinales así como sus alcances políticos ${ }^{80}$, tal como aparece en el ejemplo de Leovigildo, quien al convertirse al catolicismo (en este punto Gregorio estaría errado ${ }^{81}$ ),

75 F. L. GANSHOF, «L'historiographie dans la monarchie franque», 635. Para el tema de los pueblos bárbaros en Galia véase: W. GOFFART, Barbarians and Romans, $A$. D. 418-584. The Techniques of Accommodation (New Jersey 1980).

76 Gregorio De Tours, Historia Francorum, I, Praefatio: eclesiae inpugnarentur ab hereticis, a catholicis tegerentur.

77 GREGORIO DE TOURS, Historia Francorum, IX, 24: illa hereticae pravitatis perfidia.

78 S. TEILlET, Des goths à la nation gothique, 372-373. Véase GREGORIO DE TOURS, Historia Francorum, II, 25 VI, 18.

79 G. VInaY, San Gregorio di Tours, 22.

80 J. M. WALLACE-HADRILL, "The Work of Gregory of Tours», 59.

81 En relación a este tema Gregorio estaría mal informado, ya que es imposible dar crédito a esta afirmación hecha por el Turonense. Asimismo, como veremos más adelante, en el Chronicon de Juan de Bíclaro no se hace referencia a la conversión de Leovigildo, hecho por el cual Campos, siguiendo la opinión histórica general, niega la existencia de esta antes de morir. Cf. J. CAMPOS, Juan de Biclaro, obispo de Gerona. Su vida y su obra (Madrid 1960) 56. 
supuestamente antes de morir, orientaría en una visión favorable el gobierno de su hijo Recaredo ${ }^{82}$.

Al respecto el Turonense escribe: «Leovigildo, rey de los españoles, cayó enfermo pero, como algunos lo afirman, después de haber hecho penitencia por su error herético y rezado con fervor para que no haya nadie más que comparta esta herejía, se convirtió a la fe católica; luego de haber pasado siete días enteros llorando las iniquidades que había cometido contra Dios entregó el alma, y Recaredo, su hijo, reinó en su lugar ${ }^{83}$.

Gregorio no dejaría pasar ocasión para retomar la profesión de fe católica y, tomando como ejemplo el caso hispano, hablará de Recaredo, hijo de Leovigildo, quien después de dejar la herejía arriana para convertirse al catolicismo (589) le serviría como modelo edificante de conversión: «él creyó en Jesucristo, hijo de Dios, igual al Padre y al Espíritu Santo, y que reina por los siglos de los siglos» ${ }^{84}$.

Por lo tanto, la batalla contra la herejía daría una clave de interpretación a la Historia Francorum, la que sería importante retomar. Los herejes, unidos a su carácter de invasores, lentamente serían vencidos por el designio divino. El caso de Clodoveo resultaría ilustrador en este sentido, pues siendo un pagano convertido y bautizado en la verdadera $\mathrm{fe}^{85}$, logra vencer a Alarico en la batalla de Vouillé, cerca de Poitiers (507), lo cual se daría bajo el esquema providencial del auxilio divino: «el dicho rey Clodoveo obtuvo la victoria con la ayuda de Dios» ${ }^{86}$.

La explicación que el Turonense da del triunfo se encontraría, entonces, bajo el esquema de un rey elegido de Dios y favorecido por su conversión a la fe católica, al contrario de Alarico, quien habría perdi-

82 Cf. S. TEILLET, Des goths à la nation gothique, 380-381.

83 GREGORIO DE TOURS, Historia francorum, VIII, 46: Leuvigildus rex Hispanorum aegrotare coepit, sed, ut quidam adserunt, paenitentiam pro errore heretico agens et obtestans, ne huic heresi quisquam repperiretur consentaneus, in legem catholicam transiit, ac per septem dies in fletu perdurans pro his quae contra Deum iniquae molitus est, spiritum exhalavit. Regnavitque Richaredus, filius eius, pro eo.

84 Gregorio De TOURS, Historia Francorum, IX, 15: credidit Iesum Christum, filium Dei, aequalem Patri cum Spiritu sancto, regnantem in saecula saeculorum.

85 Ocurrido en el año 496. Cf. Gregorio de Tours, Historia Francorum, II, 31.

86 Gregorio De TOURS, Historia Francorum, II, 37: ipse rex Chlodovechus victuriam, Domino adiuvante, obtinuit. 
do su reino por seguir bajo la fe arriana ${ }^{87}$. En este sentido, la Historia Francorum actuaría también, de alguna forma, como una apología de la doctrina trinitaria formulada ya en Nicea, en contra de la herejía de su tiempo ${ }^{88}$.

Cabría recordar que para el santo obispo de Tours el triunfo y la victoria dependerían directamente de Dios, y no de los hombres ${ }^{89}$. Esto lo afirma con claridad al referirse a Childerico, de quien dice: «él ignoraba que la realización de las victorias está en la mano de Dios» ${ }^{90}$. Dios actuaría en los acontecimientos, por tanto, a través de su Providencia, tal como aparece en los relatos de la Biblia.

La intención de mostrar a hombres elegidos, como ya había hecho Eusebio de Cesarea en el caso de Constantino o incluso Teodosio, llevaría a Gregorio a retomar la figura de Clodoveo como uno más de los destinados por Dios para mostrar, a través de éxitos militares, la protección divina ${ }^{91}$, la cual iría en directa relación con la confesión de la Santísima Trinidad $^{92}$. Esta victoria sería presentada por el Turonense como el triunfo de la verdadera fe sobre la herejía, más incluso que como el triunfo de los francos sobre los godos ${ }^{93}$, lo que resaltaría la visión providencial del relato ${ }^{94}$.

Por consiguiente, las palabras de Louis Halphen resultan muy ilustradoras al momento de acercarse a lo que constituiría el sentido de la obra de

87 R. LATOUCHE (ed.), Grégoire de Tours, 14.

88 R. Morghen, «Introduzione alla lettura di Gregorio di Tours» en Gregorio di Tours. Convegni del centro di studi sulla spiritualità medievale (Perugia 1977) 18-19.

89 En cierto modo retomaría la «teología de la victoria» de Lactancio o Eusebio. Cf. B. DUMÉZIL, Les racines chrétiennes de l'Europe: conversion et liberté dans les royaumes barbares, Ve-VIIIe siècle (Paris 2005) 433.

90 Gregorio de Tours, Historia Francorum, VI, 41: nesciens, patrationem victuriarum in manu Dei consistere.

91 Clodoveo sería un Novus Constantinus. Véase: J. M. WALLACE-HADRILL, «Gregory of Tours and Bede: Their Views on the Personal Qualities of Kings» en The Early Medieval History (Oxford 1975) 98-99; CH. LELONG, Grégoire de Tours, 63; M. A. RODRÍGUEZ DE LA PEÑA, Los reyes sabios, 326.

92 L. PIETRI, «Grégoire de Tours (saint)», col. 1021.

93 S. TeILlET, Des goths à la nation gothique, 373-374, N. 40.

94 Clodoveo aparecería como «agente de la Providencia» dentro del esquema de la época. Cf. I. N. WoOD, "Gregorio de Tours y Clodoveo", La Edad Media a debate (Madrid 2003) 137. 
nuestro obispo: «el éxito de Clodoveo no puede tener duda: gracias a Dios, Alarico es derrotado y muerto; como de costumbre, si los heréticos dejan pie; el país entero es conquistado casi sin esfuerzo»" ${ }^{95}$. En otras palabras, este rey sería, a los ojos de Gregorio, un instrumento de la Providencia ${ }^{96}$.

Siguiendo en este línea, en el prólogo del libro III el obispo historiador escribe: "yo querría, si les place, comparar un poco los éxitos sobrevenidos a los cristianos que confiesan la Trinidad y los desastres sufridos por los heréticos que la dividen ${ }^{97}$. Para ello usa el ejemplo del ya citado Alarico, quien al renegar de la fe: «es privado de su reino, de su pueblo $y$, lo que es lo más importante, de la vida eterna» ${ }^{98}$. Aparecería, de esta forma, una cierta justicia que sería inminente a lo largo de la obra, y que tendría como trasfondo una suerte de teología de la historia" ${ }^{99}$, con una relación entre la tierra y el más allá ${ }^{100}$.

Otro de los casos más interesantes de la Historia Francorum es el del rey Childerico. San Gregorio tiene duras palabras para este personaje, a quien define como un blasfemo y hereje por haber impuesto doctrinas erradas en relación a la Santísima Trinidad. Tanto por sus crueldades como por la temeraria actuación que le atribuye como gobernante, no dudará en llamarlo: «el Nerón y Herodes de nuestro tiempo» ${ }^{101}$.

El rechazo a la figura del rey de Neustria (Childerico) se justificaría principalmente por su intento de imponer la herejía presionando al episcopado, tal como lo relata en el libro V. A esto sumaría, además, su política anticlerical, el haber tomado el poder por la fuerza, así como su responsabilidad en las guerras civiles ${ }^{102}$.

\footnotetext{
95 L. Halphen, «Grégoire de Tours. Historien de Clovis», 239.

96 M. REYDELLET, La royauté dans la littérature latine, 369.

97 Gregorio de TOURS, Historia Francorum, III, Prólogo: vellim, si placet, parumper conferre, quae christianis beatam confitentibus Trinitatem prospera successerint et quae hereticis eandem scindentibus fuerint in ruinam.

98 Gregorio De TOURS, Historia Francorum, III, Prólogo: Alaricus hanc denegans, a regno et populo atque ab ipsa, quod magis est, vita multatur aeterna.

99 C. MoresChINI - E. NORELl, Historia de la literatura cristiana antigua griega y latina, 596; L. PIETRI, "Grégoire de Tours (saint)», col. 1022.

100 M. REYDELLET, La royauté dans la littérature latine, 365.

101 Gregorio De TOURS, Historia Francorum, VI, 46: Chilpericus, Nero nostri temporis et Herodis.

102 CH. Lelong, Grégoire de Tours, 50.
} 
A lo anterior habría que agregar, tal como lo destaca el profesor Rodríguez de la Peña, la total reprobación por parte de Gregorio de cualquier cultivo de literatura pagana en un monarca, muy en la línea de san Gregorio Magno ${ }^{103}$. Esto se opondría a los claros intereses intelectuales de Childerico, quien contaba con un talento literario, con un saber secular y con un claro deseo de emulación de Roma y Bizancio ${ }^{104}$.

Este rey había escrito un pequeño tratado sobre la Santísima Trinidad (De Trinitate), el cual contendría algunas afirmaciones heréticas de línea arriana y sabeliana ${ }^{105}$. A esto, nuestro obispo agrega: «él sostenía que el Padre es el mismo que el Hijo e igualmente que el Espíritu Santo es el mismo que el Padre y el Hijo. Es así, decía él [Childerico] que ha aparecido en los profetas y en los patriarcas y es así que la Ley misma lo ha anunciado» ${ }^{106}$.

Después de esta declaración no resulta difícil imaginar otro motivo más para el rechazo del Turonense hacia este personaje. Asimismo, la oposición a la herejía tanto de Gregorio, en cuanto obispo de Tours, como del obispo de Albi, san Salvi, mostrarían el ascendiente del episcopado de la región en la ortodoxia frente al rey ${ }^{107}$.

Ahora bien, si la acción de la Providencia no aparecería tan claramente detallada como en el caso de Orosio, sí resultaría más o menos evidente que el proyecto de Gregorio apuntaría a una historia eclesiástica ${ }^{108}$, lo cual no se contrapondría a una historia de la situación histórica de su tiempo. Este proyecto de historia de la Iglesia, más específicamente de la Iglesia gala, sería, como ya hemos dicho, una herencia de los autores por

103 M. A. Rodríguez de la PeÑa, Los reyes sabios, 320.

104 M. A. Rodríguez de LA PEÑA, Los reyes sabios, 321.

105 M. A. Rodríguez de LA PeÑa, Los reyes sabios, 315, 320.

106 Gregorio de Tours, Historia Francorum, V, 44: adfirmans etiam, ipsum esse Patrem, qui est Filius, idemque ipsum esse Spiritum sanctum, qui Pater et Filius. 'Sic', inquid, 'prophetis ac patriarchis apparuit, sic eum ipsa lex nuntiavit'.

107 R. Latouche, «Un évêque historien: Grégoire de Tours», 258-259.

108 A. De VogüÉ, Histoire littéraire du mouvement monastique dans l'Antiquité. Première partie: Le monachisme latin de Césaire d'Arles à Grégoire de Tours (525-590), vol. IX (Paris 2005) 313-338. Este estudio, recientemente editado, es un interesante trabajo en relación a la vida monacal del siglo VI relatada por Gregorio en la Historia Francorum (se centra solo en este aspecto). Sería otro elemento más para ver la condición de historia eclesiástica de la obra. 
él utilizados ${ }^{109} \mathrm{y}$, a su vez, un planteamiento de la historia de la salvación y la evangelización en esta región ${ }^{110}$.

Estaríamos, entonces, ante una historia en la cual la acción de Dios se daría en forma particular, favoreciendo a algunos, lo cual se repetirá durante gran parte de la Edad Media. En este caso, como lo ha expresado Wallace-Hadrill, Dios aparecería en Gregorio como un Dios protector de los francos ${ }^{111}$, pero no por esto ausente de la historia universal y de sus avatares.

También, la interacción de los relatos de las virtudes de los santos con los relatos de los pueblos y de sus luchas irían unidos de tal forma que, a lo largo de los diez libros que conforman la obra, aparecerían menciones constantes a las acciones de santidad de algunos hombres. En el prólogo del libro II lo enuncia con estas palabras: «siguiendo el orden de los tiempos, exponemos mezclada y confusamente tanto los milagros de los santos como las masacres de los pueblos» ${ }^{12}$.

A lo largo de la narración se encuentran intercalados breves y compactos relatos de la justicia de Dios aplicada a través de los santos, de curaciones y otros muchos portentos ${ }^{113}$. Sin mayor conexión con el contexto de cada libro, estos aparecerían salpicando el relato y dando prueba del deseo de Gregorio de poblar su obra de un sentido religioso y, de cierta forma, edificante.

En el libro VIII, nuestro autor narra el caso de un tal Pelagio, quien es definido como un miserable que se habría enfrentado al obispo de Tours y habría abusado de los religiosos de la diócesis, situación ante la cual Dios se habría manifestado en los siguientes términos: «tan pronto como produjo la falsedad, fue tomado por la fiebre y al tercer día entregó

109 G. MONOD, Études critiques sur les sources de l'histoire mérovingienne, 121-122.

110 M. REYDELLET, La royauté dans la littérature latine, 368.

111 J. M. WALLACE-HADRILL, "Gregory of Tours and Bede», 99-100. Esta visión particular de Dios como protector de un determinado pueblo será común a los cronistas medievales.

112 Gregorio De Tours, Historia Francorum, II, Prólogo: prosequentes ordinem temporum, mixte confusequae tam virtutes sanctorum quam strages gentium memoramus.

113 A modo de ejemplo: Gregorio De TOURS, Historia Francorum, V, 6; VII, 12; VIII, 16. 
el alma» ${ }^{114}$. Relatando lo acontecido con su cuerpo, agrega: «así se manifestó la virtud de la bienaventurada María, en cuya basílica el miserable profirió un juramento falso» ${ }^{115}$.

Por lo tanto, podríamos llegar a pensar que la relación entre la hagiografía y milagros estaría mostrando, a su vez, una relación directa entre los acontecimientos de los hombres y el designio de Dios en ellos. Múltiples acontecimientos prodigiosos y vidas de mártires y de santos, todo iría unido en un relato histórico que, probablemente, no tendría otro sentido más que la creencia total de la intervención celeste en los asuntos humanos ${ }^{116}$.

Dentro del esquema de la intervención de Dios en la historia de los hombres es como se podrían entender los distintos signos de la naturaleza que aparecen a lo largo de la obra. Estos no harían más que reafirmar el hecho de que la Providencia haría suspender, en algunos momentos, el orden natural de las cosas, para así manifestar en la cotidianeidad el poder divino ${ }^{117}$.

De esta manera, los múltiples prodigios de la naturaleza que aparecen en la Historia ${ }^{118}$ tendrían una connotación profética y, a lo largo de la narración, no harían otra cosa sino mostrar el poder de Dios operante en el mundo. Dios operaría en la historia en la medida en que se haría presente, no solo a través de sus santos, sino que también a través del propio cosmos, el cual adquiriría un sentido casi mágico ${ }^{119}$.

Ahora bien, la cuestión en relación a la Providencia resulta compleja ya que, al carecer de características más bien teológicas, la Historia es-

${ }^{114}$ Gregorio De Tours, Historia Francorum, VIII, 40: in quo statim ut falcem misit, febre correptus, diae tertia spiritum exalavit.

115 Gregorio De TOURS, Historia Francorum, VIII, 40: manifesta est autem virtus beatae Mariae, in cuius basilicam miser sacramentum protullit mendax.

116 R. MORGHEN, «Introduzione alla lettura di Gregorio di Tours», 23-24.

117 G. KURTH, Études franques, 122.

118 Por ejemplo: Gregorio de Tours, Historia Francorum, IV, 31; V, 18; V, 33, entre otros.

119 R. MORGHEN, «Introduzione alla lettura di Gregorio di Tours», 18-19. A este respecto, Robert Latouche comenta que es probable que nuestro autor participara de algunas de las supersticiones de su época, pero esto no le restaría ni credibilidad ni carácter a su obra, pues correspondería a una visión común a sus contemporáneos (R. LATOUCHE (ed.), Grégoire de Tours, 14). 
crita por Gregorio no se correspondería con las interpretaciones de tipo agustinianas que se verían, por ejemplo, en Orosio. Por otra parte, este tema tendría una dificultad mayor, pues el seguimiento del historiador hispano parecería ser algo más que una simple adopción de frases o material, lo cual indicaría una visión de fondo ${ }^{120}$.

Por otra parte, para el profesor Massimo Oldoni la Historia Francorum no sería una obra providencialista. Él entiende que a lo largo de la narración: "Dios con su Providencia es lejano» ${ }^{121}$. En su concepción, el obispo de Tours sería fundamentalmente un narrador, y en este sentido las certezas, que para otros autores cristianos estarían dadas por lo trascendente, en su caso estarían marcadas por las relaciones propias de la sociedad de su época ${ }^{122}$.

Oldoni postula, a nuestro juicio erradamente, una visión entendida más bien como realista y práctica, en donde la fórmula bipolar de Orosio (expresada como Bien/Mal y concordia/discordia) ${ }^{123}$ le permitiría explicar cómo la obra no sería una compilación teológico-eclesiástica de la relación entre católicos y herejes, sino simplemente una descripción histórica con explicaciones lógicas ${ }^{124}$.

Walter Goffart escribe que la Historia Francorum no habría sido escrita en un sentido teológico, y que la relación con Dios vista en ella sería de verticalidad, es decir, un Dios ajeno a las cuestiones de los hombres. Para él la instrucción moral y el trabajo de los milagros de los santos serían el hilo conductor de toda la obra ${ }^{125}$.

Consideramos que este aspecto no resultaría necesariamente contrapuesto a una visión providencial de la historia, pues si bien Goffart plantea que Gregorio presenta la Encarnación como un hecho teológico y no tanto histórico (a diferencia de Orosio), no se podría obviar la idea del cumplimiento de las profecías en la plenitud de los tiempos ${ }^{126}$. Así tam-

\footnotetext{
120 J. M. WALLACE-HADRILL, "The Work of Gregory of Tours», 57.

121 M. Oldoni (ed.), Storia dei franchi, L.

${ }^{122}$ M. OldonI, "Gregorio di Tours e i 'Libri Historiarum'», 698-699.

${ }_{123}$ M. Oldoni (ed.), Storia dei franchi, XLI.

${ }^{124}$ M. Oldoni (ed.), Storia dei franchi, L.

125 W. GOFFART, The Narrators of Barbarian History, 205-206.

126 W. GOFFART, The Narrators of Barbarian History, p. 204.
} 
bién, si los santos obran milagrosamente, es porque un Dios providente se valdría de ellos para intervenir en la historia humana.

En nuestra opinión, la lectura de la narración histórica del obispo turonense no tendría por qué oponerse a una visión de la acción providente de Dios en el mundo. Si bien no estaríamos ante una obra en la que los presupuestos teológicos serían los fundamentales, eso no estaría demostrando la ausencia de una cosmovisión providencialista de la vida.

¿Resultaría posible entender la visión hacia Clodoveo como una simple admiración al fundador de la dinastía merovingia? Pareciera ser que no, pues las palabras del Turonense, comunes al episcopado galo ${ }^{127}$, no apuntarían sino a la idea de un hombre admirable, el cual debería contar necesariamente con el favor de Dios en tanto que convertido al catolicismo $^{128}$ : «este fue un gran hombre y un guerrero eminente» ${ }^{129}$, y a esto agrega: «el rey Clodoveo, que ha confesado, extendió su reino a través de todas las Galias» ${ }^{130}$. Habría que recordar, entonces, que para Gregorio: «el Señor restituyó el céntuplo de sus bienes a aquellos que creían verdaderamente en Él» ${ }^{131}$.

Para Wallace-Hadrill, el texto en cuestión correspondería a la tradición de la apologética histórica agustiniana ${ }^{132}$ y, por tanto, en cuanto heredero del esquema del devenir histórico marcado por san Agustín ${ }^{133}$, no podría estar ajeno a la dinámica de la acción divina en los acontecimientos de los hombres, por muy menor que esta pareciera. El carácter

127 R. LATOUCHE (ed.), Grégoire de Tours, 103, n. 48.

128 Aunque para Wood la obra de Gregorio manipularía los datos de la conversión de Clodoveo y omitiría algún tipo de acercamiento de este a la herejía, lo haría siguiendo el sentir de la Iglesia de sus días. En último caso, de ser así, estos factores estarían orientados por su propósito y por su concepción de la historia. Véase: I. N. WOOD, "Gregory of Tours and Clovis», 147, 152-153.

129 Gregorio De Tours, Historia Francorum, II, 12: hic fuit magnus et pugnatur egregius.

130 Gregorio de Tours, Historia Francorum, III, Prólogo: hanc Chlodovechus rex confessus... regnumque suum per totas Gallias dilatavit.

131 Gregorio de Tours, Historia Francorum, III, Prólogo: Dominus autem se vere credentibus,... hic centuplicata restituit.

132 J. M. WALLACE-HaDRILL, «The Work of Gregory of Tours», 70.

133 E. Mitre FernándeZ, Historia y pensamiento histórico, 56. Gregorio de Tours y los acontecimientos del reino franco se enmarcarían dentro de la sexta edad. 
narrativo no se opondría, entonces, a un carácter histórico providencial, contrario al arrianismo y orientado a destacar la Galia cristiana ${ }^{134}$.

El recuento de años hecho al final de la obra sumaría $5792^{135}$ desde la creación del mundo hasta sus días, y como un recuento cronológico, no haría otra cosa sino dejar en evidencia el orden del mundo a partir de una visión de fe. Una de las fechas claves sería la muerte de san Martín, acontecida 412 años después de la Resurrección del Señor; otro dato importante para ver el enfoque eclesial que habría querido dar a su obra ${ }^{136}$.

Por consiguiente, la hagiografía y los milagros no serían solo un recurso dentro del texto, ni tampoco actuarían como el gran motor de los acontecimientos, sino que estos corresponderían al esquema de los hombres justos a través de los cuales operaría la acción de Dios. ¿No sería esta una forma de acción de la Providencia en la historia humana? Tal vez se podrían repetir las palabras de la Historia Francorum en donde su autor recuerda la centralidad de lo trascendente: «cuando se merece tener un remedio celeste no deben buscar cuidados terrestres» ${ }^{137}$.

Si bien Gregorio, a partir del libro V, sigue los hechos del reino franco desde su propia experiencia, el resumen de la historia bíblica que se encuentra a lo largo del libro I no haría sino remitir al lector a la historia sagrada ${ }^{138}$, dándole un marco universal. Luego, aunque la mayor parte de la obra relata anécdotas y situaciones coyunturales del reino en cuestión, el poco conocimiento en profundidad del mundo bárbaro se podría explicar por su interés en la historia eclesiástica de su época ${ }^{139}$.

Otro aspecto importante de analizar en el santo de Tours es el lugar dado al nacimiento de Jesucristo. Como hemos visto, resulta indudable que para toda teología cristiana de la historia este es el punto axial de la historia de la humanidad. Por esto ha sido cuestionado el lugar que

134 R. MORGHEN, «Introduzione alla lettura di Gregorio di Tours», 18-24.

135 Gregorio De TOURS, Historia Francorum, X, 31: quorum omnis summa est anni VDCCXCII. Véase R. LATOUCHE (ed.), Grégoire de Tours, 10. Puede resultar útil la lectura del comentario de la introducción (8-11).

136 Cf. M. REYDELLET, La royauté dans la littérature latine, 368.

137 Gregorio de Tours, Historia Francorum, V, 6: quando caelestem accipere meruerit medicinam, terrena non requirat studia.

138 F. L. GANSHOF, «L'historiographie dans la monarchie franque», 633-634.

139 P. RICHÉ, Éducation et culture, 226. 
este acontecimiento adquiriría en la obra del Turonense. Para Goffart, la descripción de nuestro autor haría de este episodio un hecho teológico fundamental, pero no habría relación con la centralidad que Orosio daría a este dentro del Imperio Romano ${ }^{140}$.

En el libro I de la Historia el autor escribe: «en el año 43 del reino de Augusto nuestro Señor Jesucristo nació según la carne como lo hemos dicho, de la Virgen María, en Belén, fortaleza de David ${ }^{141}$. Si bien es la única mención al nacimiento de Jesús, este simple hecho le bastaría, ya que el relato bíblico sería conocido ampliamente por todos, más si su público lector estaba conformado mayoritariamente por clérigos ${ }^{142}$.

No creemos que se pueda llegar a afirmar la carencia de un sentido providencial de la historia por el enfoque dado a un acontecimiento que, en tanto que fundamental, se entendería como parte del conocimiento bíblico de cualquier letrado de su tiempo. Por otro lado, aunque con connotaciones distintas, en esto veríamos una similitud con Sulpicio Severo, quien por respeto a la Sagrada Escritura hará breves menciones de los sucesos principales de la fe cristiana, como por ejemplo la Natividad del Señor.

Por lo tanto, la fe de Gregorio de Tours debería ser el punto de partida para una lectura atenta de su Historia Francorum y, en ella, firmemente enraizada, la concepción de la Providencia como guía de los acontecimientos de la historia. Si bien no estaríamos ante un autor con características de teólogo, no podríamos dar por supuesto que la teología de la historia no estaría en la base de su narración histórica, lo cual no se opondría a la posibilidad de llamarlo: «el Heródoto de la barbarie» ${ }^{143}$.

Finalmente, habría que destacar que su confianza absoluta en el poder de Dios y en la acción de los santos, su ortodoxia y fidelidad a la doctrina, así como su compromiso con la labor de pastor de la Iglesia

140 W. GOFFART, The Narrators of Barbarian History, 204.

141 Gregorio de TOURS, Historia Francorum, I, 19: Anno XLIII imperii Agusti dominus noster Iesus Christus, ut diximus, ex virgine Maria in Bethleem David oppidum secundum carnem natus est.

${ }_{142}$ P. ANTIN, «Emplois de la Bible chez Grégoire de Tours et Mgr Pie», 780. En esta época todavía había un público laico, aunque en claro retroceso.

${ }_{143}$ G. MonOD, Études critiques sur les sources de l'histoire mérovingienne, 114. 
de Tours, desempeñada con vigilancia y seria actividad ${ }^{144}$, harían de él y de su obra histórica un conjunto que difícilmente podría llegar a entenderse sin comprender las profundas convicciones religiosas que estarían tras su relato.

Nos parece que, a pesar de todas sus limitaciones, el espíritu sensible y siempre alerta ${ }^{145}$ de Gregorio permitiría al lector retomar las autorizadas y, a nuestro juicio, actuales palabras de Gabriel Monod escritas a finales del siglo XIX: «a pesar de su ignorancia, a pesar de la desgracia de los tiempos, a pesar de las agitaciones y de las ocupaciones infinitas, él se impuso aún como un deber el componer diez libros de historia [...] para la edificación de sus hermanos y la instrucción de la posteridad ${ }^{146}$.

${ }^{144}$ CH. LELONG, Grégoire de Tours, 69-78.

145 Ch. Lelong, Grégoire de Tours, 78.

146 G. MONOD, Études critiques sur les sources de l'histoire mérovingienne, 123. 
Resumen: San Gregorio de Tours es un autor galorromano que vivió una época de confusión y turbulencias a causa de las invasiones bárbaras y las herejías. Con una formación centrada fundamentalmente en las Sagradas Escrituras, él se propuso mostrar la historia de su tiempo y la intervención de Dios en ella a través de los milagros. Su principal obra, la Historia de los francos (Historia Francorum), se enmarca dentro de la dinámica cristiana de la «teología de la historia». Aunque en ella se evidencia una visión más bien local, también se encuentra presente un sentido universal cristiano. Su relato se constituye en un texto de capital importancia para comprender el cambio desde la concepción imperial romana hacia nuevas realidades históricas particulares, en este caso, el mundo de los francos. Con un fin pastoral y moralizador, este obispo de Tours aborda la acción de la Providencia en la historia -especialmente a través de la hagiografía-, así como el problema de la necesaria conversión de los individuos y de los pueblos.

Palabras Clave: Teología de la historia, Providencia, milagros, Gregorio de Tours, conversión.

Abstract: The Gallo-Roman author Saint Gregory of Tours lived in a period of chaos and turbulences caused by the Barbarian invasions and the current heresies. With a formation mainly centered in the Scriptures, his proposal was to prove God's intervention in the history of his time through His miracles. His main work, the Historia Francorum is framed inside the Christian dynamics of the 'theology of history'. Although it evidences a more less local vision, it is also present a Christian universal sense. His narration has become a text of capital importance for the best understanding of the change from the Roman imperial vision to new historical realities; in this particular case, the world of Francs. This Bishop of Tours studies Providence action through history -especially through hagiography- with an objective both pastoral and moralizer, and, with a similar goal, the problem of the necessary conversion of individuals and societies.

Keywords: Theology of history, Providence, miracles, Gregory of Tours, conversion. 